\title{
Corea del Sur ante la crisis financiera global: costos vs. beneficios de la apertura financiera
}

DOI: $10.32870 /$ mycp.v12i36.331

Geneviève Marchini ${ }^{1}$

L os primeros años del nuevo siglo han sido favorables para el crecimiento económico de las economías emergentes y en desarrollo, pues pasada la etapa de crisis y reformas estructurales dolorosas, se han beneficiado de la fuerte demanda en las economías desarrolladas, en especial Estados Unidos, la cual ha sostenido la expansión de sus exportaciones. En un contexto de abundante liquidez internacional, estos desarrollos les permitieron mejorar sus razones de endeudamiento externo y sus indicadores de solvencia. En Asia del Este, históricamente más orientada hacia los mercados internacionales, la recuperación de la crisis de 1997-1998 ha ido de la mano del dinamismo de las exportaciones manufactureras y de masivas entradas de capitales externos que han complicado el manejo macroeconómico, sin que su contribución a la inversión productiva sea destacada, pues el "dinero caliente" constituyó una elevada proporción de estos flujos. Corea del Sur (en lo que sigue, Corea) no ha sido la excepción: desde 1998 y hasta 2007 la economía ha presentado superávits en cuenta corriente, a la vez que recibía flujos netos de fondos a altura de $2 \%$ del PIB en promedio, una sobreabundancia que impulsó una apreciación sostenida de la moneda nacional aun cuando las autoridades han acumulado reservas internacionales a niveles nunca vistos en el país.

A partir de 2007, la crisis de las hipotecas de alto riesgo señaló las bases insostenibles del auge económico mundial, y su transmisión a otros mercados

1. Profesora-investigadora del Departamento de Estudios del Pacífico, Centro Universitario de Ciencias Sociales y Huanidades de la Universidad de Guadalajara. 
y a otras regiones del mundo empezó a revertir los anteriores efectos sobre las economías emergentes y en desarrollo. En sus inicios, el impacto directo del desplome de la burbuja financiera sobre estas economías sólo fue moderadamente negativo, pues sus bancos e instituciones financieras se hallaban poco expuestos a los activos tóxicos estadounidenses. Fue más bien en el tercer trimestre de 2008 que estas economías fueron contagiadas, cuando cundió el pánico financiero internacional producido por la debacle del banco de inversión Lehman Brothers, y se dieron simultáneamente una aguda contracción del crédito y una reversión de los flujos de capitales. Corea experimentó salidas moderadas de capitales de su mercado accionario desde los inicios de la crisis del crédito subprime, en 2006, seguidas de salidas masivas en 2007 cuyo impacto fue compensado por fuertes entradas de fondos bancarios, pero fue sólo desde mediados de 2008 que sufrió un deterioro pronunciado de su economía real. Éste empezó con una disminución moderada de sus exportaciones de bienes, que se transformó a partir de septiembre en una caída estrepitosa, combinada con salidas masivas de capitales externos y con una aguda pérdida de valor de su moneda, el won. Hechos similares se produjeron con pocas excepciones en las economías emergentes de Asia, América Latina y Europa del Este, e incluso en naciones desarrolladas muy dependientes del financiamiento externo, como Australia y Nueva Zelanda.

A pesar de sus profundas reformas posteriores a la crisis asiática, que han procurado volver su sistema financiero más resistente a los choques externos, y de su buen desempeño macroeconómico, la economía de Corea ha sido golpeada por el pánico del último trimestre de 2008 con más fuerza que otras economías vecinas del Este de Asia, pues de nuevo se puso en duda su capacidad de servir sus pasivos financieros externos. En este artículo se propone analizar la vulnerabilidad financiera de Corea en la crisis de 20072008; en particular se plantea establecer a través de qué canales se produjo el contagio del país, si éste respondió a debilidades específicas de su esfera financiera interna, y en qué medida se derivó de la posición de Corea en tanto mercado financiero emergente en la esfera financiera global. El artículo se ubica en el marco de una perspectiva crítica de las políticas de apertura financiera adoptadas nolens volens por una mayoría de economías emergentes y en desarrollo desde la década de 1990. Plantea que las reformas financieras coreanas han logrado fortalecer la banca y la esfera financiera del país, resolviendo las debilidades puestas en evidencia por la crisis de 1997. A la vez, han surgido nuevas fuentes internas de riesgos financieros, y el carácter 
financieramente más abierto de Corea ha vinculado más estrechamente los mercados financieros locales a los desarrollos de la esfera financiera global. Los masivos flujos de carácter especulativo que ha recibido el país, si bien han estimulado el desarrollo de los mercados financieros locales, contribuyeron poco a la inversión productiva y crearon externalidades negativas para la economía, al fortalecer nuevos canales de contagio y elevar su vulnerabilidad financiera externa.

El plan del artículo es como sigue: se hace en una primera sección un breve recuento de las transformaciones introducidas en las políticas económicas y en la economía coreana después de la crisis asiática, con un objetivo general de mejor adaptación y mayor solidez frente la economía global y se evalúan sus efectos en la banca. En la segunda sección se analizan aspectos centrales de la vulnerabilidad externa de la economía coreana en 2008, y en la tercera se introduce el contagio de Corea por la crisis internacional y se reflexiona acerca de los riesgos y beneficios de la apertura de la cuenta de capitales de la balanza de pagos para una economía como la coreana. En la última sección se concluye.

\section{De 1997 a 2008: reformas estructurales y políticas económicas de una crisis a otra ${ }^{2}$}

En 1997 Corea del Sur se hallaba en medio de un proceso de liberalización económica, iniciado en los años 1980 y acelerado a partir de 1993, que volvió su economía más vulnerable a una crisis financiera externa. A pesar del carácter paulatino y pragmático de las reformas financieras coreanas, que priorizaron la estabilidad y el desempeño de la economía real, la transición en curso desde el modelo de crecimiento basado en el alto endeudamiento empresarial con garantía pública implícita, que le permitió desarrollarse rápidamente desde la década de $1960^{3}$ hacia una economía de libre-mercado comercial y

2. En parte de esta sección se retoman aportaciones de un trabajo anterior; véase Marchini, Genevieve, "Reformas financieras y crisis bancaria en Corea del Sur", Problemas del desarrollo, vol. 31, núm. 123, octubre-diciembre 2000, México, pp. 79-105, para un análisis más pormenorizado.

3. Wade, Robert, y Frank Veneroso, "The Asian Crisis: the High Debt Model vs. the Wall StreetTreasury-IMF Complex", New Left Review, marzo-abril de 1998. 
financieramente más abierta, creó un cuadro de vulnerabilidad financiera, ${ }^{4}$ producto de la puesta en contacto de un sistema financiero interno frágil y de la sobreabundancia y la volatilidad de los flujos de capitales externos que la economía atrajo a partir de 1993. La liberalización financiera coreana, definida de manera bastante autónoma y pragmática, evitó muchos de los escollos sobre los cuales naufragaron las reformas financieras "de choque" introducidas en América Latina bajo la tutela de los organismos financieros multilaterales. En primer lugar, la secuencia de reformas económicas que se inició en 1980 privilegió la apertura comercial y una diversificación institucional del sistema financiero interno al abrigo de controles de capitales, mientras el sistema bancario, privatizado pero estrechamente controlado por las autoridades, proveía las empresas con créditos a tasas administradas. En segundo lugar, Corea, que recurrió a un endeudamiento internacional controlado para financiar su crecimiento económico de posguerra, inició un prudente proceso de apertura financiera, limitado inicialmente a una liberalización parcial de las salidas de capitales coreanos en un contexto de superávits en cuenta corriente (finales de la década de 1980).

Sin embargo, la aceleración de las reformas financieras a inicios de los años 1990 - asociada al esfuerzo de Corea por entrar a la OCDE- hizo coincidir la liberalización del sistema bancario interno, largamente postergada debido al papel estratégico que desempeñaba en el modelo de desarrollo coreano, ${ }^{5}$ y una apertura asimétrica de la cuenta de capitales a flujos provenientes del exterior: Corea abrió parcialmente sus mercados accionarios a las inversiones de portafolio, siguió restringiendo el acceso a los mercados de bonos y a la inversión extranjera directa (IED) y liberalizó la contratación de deudas (préstamos y bonos) internacionales de corto plazo de parte de las instituciones bancarias locales, mientras el endeudamiento externo de las corporaciones seguía sujeto a límites. Como consecuencia, los flujos de portafolio hacia

4. Utilizamos la definición propuesta en Gavin, Michael, y Ricardo Asuman, "Les origines des crises bancaires: le contexte macroéconomique”, Problèmes d’Amérique Latine, núm. 21, abriljunio de 1996: 117-147, que plantea que existe vulnerabilidad financiera cuando "pequeños choques al ingreso, calidad de los activos o la liquidez de una entidad financiera la vuelven insolvente o suficientemente ilíquida como para no poder atender sus compromisos financieros de corto plazo".

5. Dos clases de medidas destacan por su importancia: la desregulación de las tasas de interés pasivas y activas, y la liberalización de las operaciones bancarias, que permitió a los bancos competir con las instituciones financieras no bancarias (IFNB) con productos menos regulados. A la vez, parte de las IFNB accedieron a la categoría de merchant banks. 
el mercado accionario y el endeudamiento externo de la banca coreana se volvieron los vehículos privilegiados para la entrada de capitales externos en el país. Entre 1993 y 1996 la banca comercial y los merchant banks (banca de inversión) - el brazo financiero de los conglomerados-cuadruplicaron sus pasivos externos, utilizándolos en parte para alimentar la oferta de crédito interno a las empresas. ${ }^{6}$ La transformación de vencimientos operada por los bancos a partir de estos fondos en divisas - deudas a corto plazo y préstamos a mediano o largo plazos-y la alta exposición del sistema en su conjunto a los chaebol, combinados con las carencias de la reglamentación y de la supervisión prudencial, potenciaron los riesgos inherentes a la contratación de préstamos internacionales denominados en divisas y elevaron la vulnerabilidad de la economía coreana a choques negativos provenientes de la economía internacional.

En 1996-1997 las empresas coreanas habían aprovechado el mayor acceso al financiamiento para seguir un proceso agresivo de expansión que llevó a la sobreinversión en varios sectores exportadores, y elevó todavía más su endeudamiento relativo en comparación con los niveles muy altos que prevalecían a finales de los ochenta; por ello presentaban la razón de deuda a capital más elevada de Asia del Este (3.5 veces). A la vez, si bien la garantía pública implícita de los proyectos empresariales no había sido denunciada formalmente, el creciente endeudamiento en divisas limitaba en los hechos la capacidad de rescate de parte del gobierno coreano. En el plano macroeconómico, la vulnerabilidad financiera externa de la economía era puesta en evidencia por el alto nivel de sus deudas de corto plazo, que duplicaban el valor de las reservas internacionales, y a partir de 1996, por un amplio déficit en cuenta corriente ( $4.7 \%$ del PIB) asociado a un deterioro del valor de sus productos de exportación líderes. La banca coreana, principal intermediaria de los flujos de capitales que entraron al país, y muy expuesta a los chaebol, estuvo en el epicentro de la crisis de 1997: el deterioro de las ventas internacionales de conglomerados altamente endeudados y las quiebras empresariales de la primera mitad del año, antecedieron la estampida de los flujos de capitales y la crisis de balanza de pagos de octubre de 1997, cuando se registraron salidas netas de fondos bancarios por cerca de 5\% del PIB. Ambos eventos materializaron los riesgos a los cuales el sistema bancario se hallaba expuesto,

6. Parte de los fondos fueron también dedicados a inversiones fuera del país — con frecuencia de alto riesgo- operadas por las filiales offshore de los bancos. 
así como los límites de la capacidad de rescate financiero del Estado coreano en un contexto financieramente más abierto, donde el papel de prestamista de última instancia del banco central se halla limitado por la disponibilidad de reservas de divisas.

La respuesta del gobierno coreano a la crisis, que se dio en el marco de un plan de ajuste financiado por el Fondo Monetario Internacional, consistió en introducir más ortodoxia económica, tratando de esta manera de atender las debilidades señaladas por los análisis de las instituciones financieras multilaterales, que achacaron principalmente la crisis asiática a las fallas de los mercados financieros internos y de la regulación y supervisión prudencial nacional de las economías de la región. Las autoridades coreanas decidieron profundizar la liberalización financiera externa, a la vez que aceleraban la reforma del modelo económico para resolver los aspectos generadores de vulnerabilidad financiera, en particular el alto endeudamiento empresarial, la elevada exposición del sistema financiero a la deuda de los conglomerados, y las fallas del marco prudencial. Así, las reformas incluyeron una reestructuración empresarial y financiera conjunta emprendida bajo la tutela estatal, una modificación de las relaciones Estado-empresas-bancos que significó la desaparición de elementos centrales del modelo económico de posguerra, y el fortalecimiento del marco regulatorio y de la supervisión. Por su lado, la profundización de la apertura financiera incluyó la flexibilización de la inversión extranjera directa (IED) en el país, la apertura plena del mercado accionario y de los mercados de bonos a la inversión de portafolio y la liberalización del mercado de cambios. Corea abandonó el régimen de tipo de cambio fijo y adoptó un tipo de cambio flexible, a la vez que una política monetaria basada en metas de inflación.

Ciertamente este conjunto de medidas ha logrado disminuir los riesgos que existían en 1997 en el sistema bancario interno, que ha enfrentado la crisis de 2008 en condiciones más sólidas que en 1997, como lo señalan sus niveles de capitalización más altos, sus buenos indicadores de rentabilidad y la baja proporción de préstamos de mala calidad (cuadro 1), si bien nuevas fuentes de riesgo - el sobreendeudamiento de los hogares y el creciente recurso de los bancos al fondeo en el mercado de mayoreo- han aparecido. En primer lugar, el sistema bancario se ha consolidado pues han desaparecido las instituciones más débiles y menos reglamentadas - a través de fusiones o cierre, los bancos comerciales pasaron de 33 a 18 entre 1997 y 2006, y sólo 
quedaban dos merchant banks en operación en 2006, contra 30 en 1997_, ${ }^{7}$ las oportunidades de "arbitraje" reglamentario más visibles han desaparecido y las instituciones sobrevivientes se hallan mejor capitalizadas y supervisadas. En segundo lugar, la reforma corporativa, aun cuando se considera incompleta, ha logrado disminuir el endeudamiento de las empresas coreanas, en especial de las más grandes, que presentaban en 2007 razones de deuda/capital considerablemente inferiores a sus niveles históricos (la razón de deuda a capital era de 99\% en 2006). ${ }^{8}$ En tercer lugar, este menor apalancamiento corporativo ha ido asociado a una disminución de la exposición del sistema a los chaebol, ya que los bancos comerciales orientaron sus recursos principalmente hacia los hogares y las pequeñas y medianas empresas (pymes). En cuarto lugar, se ha fortalecido dramáticamente el grado de control extranjero de la banca comercial de menudeo, y esta consecuencia notable de la reestructuración financiera y de la apertura del sistema ha sido considerada en los círculos financieros internacionales como un elemento modernizador y estabilizador, que contribuiría a elevar el estatus internacional y reducir el nivel de riesgo del conjunto del sistema bancario doméstico. Los bancos de alcance nacional, que representan $65 \%$ de los activos del sistema, se hallan ahora dominados por intereses extranjeros que han contribuido a cambiar las reglas del juego y a establecer relaciones más distantes entre bancos y autoridades y entre bancos y conglomerados. ${ }^{9}$ Una tendencia similar se observa en las demás instituciones financieras - aseguradoras, compañías de gestión de activos, casas de títulos-, donde en promedio 38\% de las entidades mostraban una participación extranjera que superaba $50 \%$ de su capital accionario. ${ }^{10}$

7. Datos de 2008 del Bank of Korea.

8. FMI, Country Report núm. 08/297, 2008. Por otro lado, la situación financiera de las pequeñas y medianas empresas, si bien ha mejorado en su conjunto desde 1997, sigue constituyendo un "punto negro" para la economía coreana y un elemento que pone en riesgo la recuperación económica poscrisis.

9. Antes de 1997 ya estaban presentes numerosas sucursales de bancos extranjeros en Corea, que operaban con restricciones en el mercado bancario de mayoreo o en los mercados de divisas. La inversión extranjera ha penetrado en el sistema de banca de menudeo de dos maneras: en primer lugar, ha adquirido paquetes accionarios mayoritarios en las instituciones intervenidas y saneadas por las autoridades cuando éstas han decidido privatizarlas de nuevo (son los casos de Citibank, de Standard Chartered y de KEB), y en segundo lugar ha comprado participaciones minoritarias en la bolsa de Seúl. En 2007 el capital de estos bancos se hallaba $55 \%$ en manos extranjeras.

10. Kim, Kyungsoo, Byoung-Ki Kim, y Young Kyung Suh, "Opening to Capital Flows and Implications from Korea”, Working Paper, núm. 363, Institute for Monetary and Economic Research, Bank of Korea, febrero de 2009: 7. 


\section{Cuadro 1}

Corea del Sur: indicadores de solidez financiera, 1999-2007

\begin{tabular}{|c|c|c|c|c|c|c|c|c|c|}
\hline & 1999 & 2000 & 2001 & 2002 & 2003 & 2004 & 2005 & 2006 & 2007 \\
\hline Crédito total/PIB (\%) & 111.6 & 107.4 & 103.6 & 119.5 & 97.8 & 94.2 & 98.1 & 109.7 & 119.2 \\
\hline \multicolumn{10}{|c|}{ Indicadores del sistema bancario } \\
\hline Razón de capitalización & 11.9 & 10.6 & 11.7 & 11.3 & 11.2 & 12.1 & 13 & 12.8 & 12.3 \\
\hline Razón de capital "Tier 1" & 7.7 & 6.7 & 7.7 & 7.2 & 7 & 8 & 9.3 & 9.2 & 8.9 \\
\hline $\begin{array}{l}\text { Rendimiento/activos } \\
\text { ("ROA") }\end{array}$ & -0.8 & -0.6 & 0.7 & 0.6 & 0.2 & 0.9 & 1.3 & 1.1 & 1.1 \\
\hline Margen de interés neto & 2.5 & 2.4 & 2.6 & 2.7 & 2.6 & 2.6 & 2.8 & 2.6 & 2.4 \\
\hline $\begin{array}{l}\text { Créditos dudosos } \\
\text { (\% del total) }\end{array}$ & nd & nd & 3.3 & 2.4 & 2.8 & 2 & 1.3 & 0.9 & 0.7 \\
\hline (bancos comerciales) & & & & & & & & & \\
\hline \multicolumn{10}{|c|}{ Sector corporativo } \\
\hline Razón deuda/capital & 214.7 & 210.6 & 182.2 & 135.4 & 123.4 & 104.2 & 100.9 & 98.9 & nd \\
\hline Razón deuda/ventas & 52.8 & 42.7 & 40.1 & 29.8 & 26.6 & 20.7 & 19.9 & 19.9 & nd \\
\hline \multicolumn{10}{|l|}{ Hogares } \\
\hline $\begin{array}{l}\text { Crédito a hogares } \\
\text { (\% del PIB) }\end{array}$ & 38.4 & 45.4 & 54.5 & 62.6 & 59.8 & 60.1 & 62.6 & 67.5 & 68.3 \\
\hline
\end{tabular}

nd: no disponible

Fuente: FMI, 2008 y 2009.

Ahora bien, en una repetición de los efectos de la liberalización financiera en las economías desarrolladas, en especial anglosajonas, la evolución del sistema bancario liberalizado ha creado nuevos riesgos: en primer lugar, los bancos, encabezados por los bancos extranjeros, y las instituciones financieras no bancarias, compensaron el declive de la demanda de crédito corporativo elevando agresivamente su oferta de crédito a los hogares - a través de crédito al consumo, de crédito hipotecario y de tarjetas de crédito-, creando en pocos años un fenómeno de sobreendeudamiento entre las familias coreanas, a la vez que una burbuja en el mercado inmobiliario residencial. Como resultado, el endeudamiento de los hogares coreanos superaba $80 \%$ del PIB en 2007, un nivel que les sitúa entre los más endeudados del mundo. ${ }^{11}$ Aun cuando la deuda hipotecaria de los hogares no presenta un nivel de riesgo sistémico elevado,

11. La deuda de las familias coreanas supera la observada en Japón (más de $60 \%$ del PIB) y es menor que la registrada en Australia, Estados Unidos y el Reino Unido, donde es aproximadamente de 100\% del PIB (FMI, Country Report, núm. 09/262, 2009: 20. 
pues sólo representa alrededor de $30 \%$ del PIB, con una relación entre el valor del préstamo y el valor del inmueble de $50 \%$, un nivel bastante conservador frente a lo observado en las economías anglosajonas, y una proporción moderada del servicio de esta deuda en relación con el ingreso de los acreditados $(20 \%),{ }^{12}$ la presencia de una elevada deuda de consumo de cerca de $50 \%$ del PIB está en el origen de una situación de sobreendeudamiento no resuelta entre las familias coreanas. ${ }^{13}$ Simultáneamente, el ahorro personal neto se ha desplomado, cayendo de un nivel promedio de $19 \%$ del PIB en la década de 1990 , a menos de $5 \%$ en promedio desde $2000 .{ }^{14}$ En segundo lugar, el crecimiento más lento de los depósitos bancarios de los hogares - parte de una diversificación de sus inversiones financieras - ${ }^{15}$ empujó a los bancos coreanos hacia la colocación de títulos negociables (certificados de depósito - $\mathrm{CD}$-, bonos bancarios) en los mercados de mayoreo - un financiamiento más caro y volátil que los depósitos-, para fondear la expansión de sus actividades internas, en particular en 2006-2007; la razón de préstamos a depósitos se ubicaba en 2007 cercano a 1.15, la segunda más alta de una muestra de economías de Asia-Pacífico, después de Australia. ${ }^{16}$ Aun cuando son sobre todo los bancos extranjeros que operan en Corea quienes se encuentran detrás de esta evolución, este hecho se convirtió en un importante factor de riesgo en la crisis de liquidez internacional del cuarto trimestre de 2008.

12. Kim, Kyungsoo, Byoung-Ki Kim, y Young Kyung Suh, "Opening to Capital Flows and Implications from Korea", op. cit., p. 34.

13. En contraste con el pico de la crisis de 2003, cuando 3.72 millones de deudores tenían retrasos de más de tres meses en pagos superiores a 500,000 wons, 2.7 millones de personas se encontraban en esta situación en junio de 2007 y cerca de siete millones de coreanos presentaban un récord de crédito deteriorado (Financial Times, “South Korea's new regime aims to stave off consumer credit crisis", 04/01/2008).

14. La tasa promedio de ahorro personal neto había descendido de $21 \%$ en $1990-1994$ a $17.6 \%$ en 1995-1999, y en 2000 se situaba en menos de $10 \%$ del PIB.

15. Las compañías de gestión de activos, que ofrecen la posibilidad de invertir en activos externos, han atraído las inversiones financieras de los hogares coreanos: los fondos depositados en estas compañías constituían $27 \%$ de los depósitos totales de las familias coreanas — suma de depósitos bancarios y depósitos en compañías de gestión de activos- en 2007 (Ahn, Byung Chan, "Capital flows and effects on financial markets in Korea: developments and policy responses", BIS Papers, núm. 44, Basilea, 2000: 313). Por otro lado, se estima que $15 \%$ de los activos financieros de los hogares se encontraban invertidos en acciones en 2008 (FMI, Country Report, núm. 09/262, 2009, op. cit., p. 11).

16. McCauley, Robert, y Jens Zukunft, "Asian banks and the international interbank market", BIS Quarterly Review, junio de 2008: 67-79 (p. 71). 


\section{Apertura y vulnerabilidad externa de la economía coreana en 2008}

En el plano macroeconómico los resultados de estas políticas afines a la ortodoxia del Consenso de Washington son mixtos: por un lado, la recuperación de la economía coreana ha sido rápida después de 1997-1998, y su desempeño ha sido bastante honorable hasta 2008. Por otro lado, desde inicios de 2000 la economía ha mostrado ritmos de inversión y de crecimiento menores a los que predominaban antes de la crisis (gráfica 1), y su vulnerabilidad externa permanece, al crearse nuevas fuentes de fragilidad asociadas a la posición del país en la globalización financiera. El menor nivel de la formación bruta de capital fijo en relación con el PIB se explica en buena parte porque las empresas manufactureras coreanas han dirigido una proporción creciente de su inversión hacia China, donde han localizado parte de su producción: ${ }^{17}$ indudablemente, la sostenida apreciación del won de 2002 a 2007 y la liberalización de la inversión directa coreana en el exterior, tomada en respuesta a ésta, han adelantado y acelerado este proceso irreversible asociado a la "maduración" de la economía coreana. A la vez, el crecimiento ha sido menos equilibrado, pues se ha logrado sobre la base de una mayor dependencia de la demanda externa. En efecto, la ampliación de la demanda final privada, que había contribuido de manera decisiva a la rápida recuperación de la economía entre 1998 y 2003, se ha frenado fuertemente en los años posteriores debido a que respondió a un proceso de endeudamiento insostenible de los hogares coreanos. Así, en el último lustro las exportaciones netas y la inversión privada, estrechamente vinculada a éstas, han explicado una mayor parte del crecimiento, frente al estancamiento de las demás fuentes internas de demanda. La combinación de débil consumo interno y de fuerte dinamismo exportador acentuó el carácter extrovertido de la economía coreana, como lo muestra la razón de exportaciones/PIB, que pasó de 32\% en 1997 a cerca de $46 \%$ en 2007, volviéndola más vulnerable a choques negativos que afecten a la demanda externa (gráfica 2), y explica el superávit duradero de la balanza comercial y de la cuenta corriente, que se han mantenido durante todo el periodo, exceptuando 2008 para la última.

17. Lim, Kyung-Mook, y Wonhyuk Lim, "Investment Bust in Post-Crisis Korea: Fact or Fiction?", Asian Economic Papers, vol. 5, núm. 3, 2007: 1-24. 


\section{Gráfica 1}

Corea del Sur: inversión y crecimiento del PIB, 1990-2007

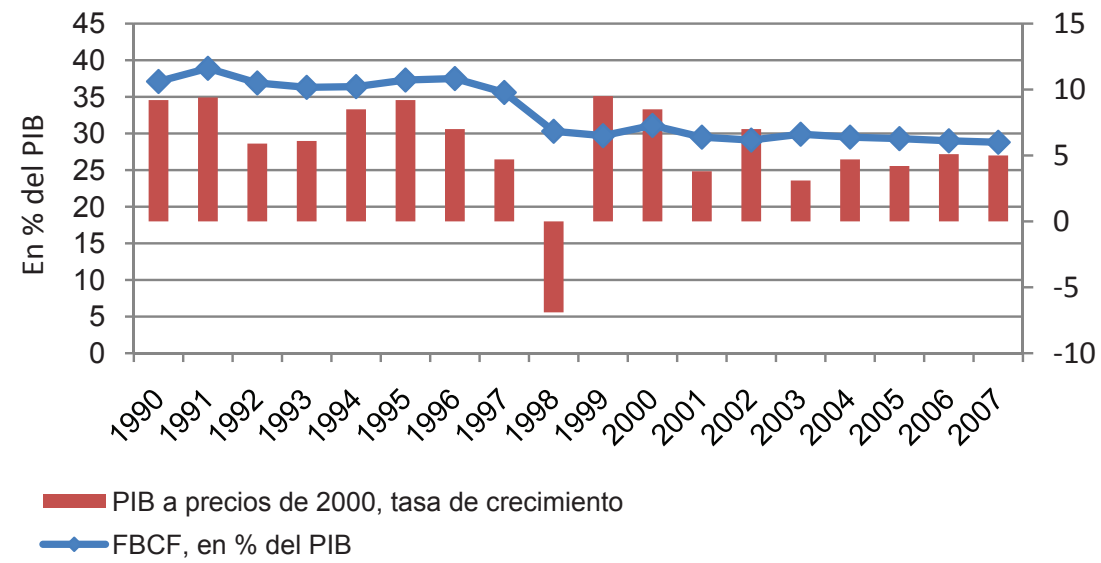

Fuente: Bank of Korea

Por otro lado, si bien las exportaciones coreanas se han diversificado en la última década, tanto en cuanto a productos como en sus destinos, ${ }^{18}$ siguen dependiendo en buena medida, tanto directa como indirectamente, de la demanda final de bienes de tecnología avanzada, y media de parte de los grandes mercados desarrollados como Estados Unidos, la Unión Europea o Japón. Por ello, las exportaciones han empezado a declinar desde julio de 2008 debido al debilitamiento de esta demanda, desplomándose en el último trimestre a un ritmo anualizado de -30\% — superior al de la crisis de 2001-, en todos los mercados, cuando a la caída de la demanda de bienes se agregó la severa contracción del crédito comercial. Este choque "real" paralizó el principal motor de la economía coreana, y tanto el consumo privado como el PIB se han contraído, este último a un ritmo anualizado cercano a $20 \%$ en el último trimestre de 2008.

18. Las exportaciones directas de Corea hacia los mercados desarrollados se han contraído, debido a la pérdida de competitividad de Corea frente a China en bienes más intensivos en mano de obra. Sin embargo, la exposición indirecta a estos mercados ha aumentado, a través del papel de proveedor y de inversionista directo de Corea en este país. 
Gráfica 2

Exportaciones e importaciones, 1990-2007

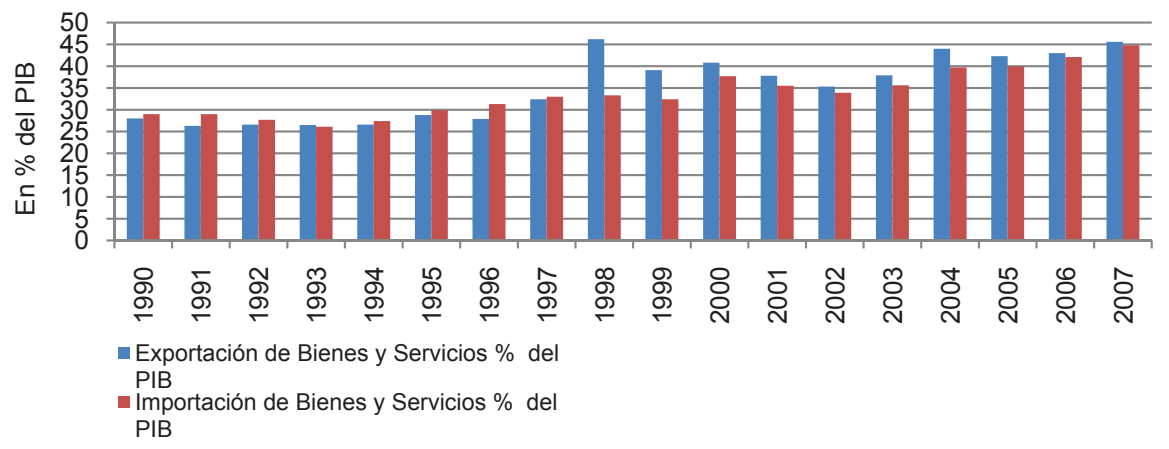

Fuente: Bank of Korea

Ahora bien, a pesar de la severidad de la caída de la demanda de bienes, es la reversión de los flujos de capitales y su impacto desestabilizador que han repercutido en forma más negativa sobre la economía coreana. En efecto, desde 1999 Corea ha vuelto a recibir masivos flujos de capitales externos tanto en términos brutos como a nivel neto. Las entradas brutas de fondos, de 23.5 mil millones de dólares estadounidenses en promedio entre 2002 y 2005 , se elevaron a 59.5 mil millones y 84.7 mil millones en 2006 y 2007 respectivamente ${ }^{19} \mathrm{o}$, en proporción del PIB, a 6.7 y $8.7 \%$, un nivel superior a lo observado durante los años que antecedieron la crisis de 1997 (gráfica 3).

La magnitud de los flujos brutos recibidos por el país, en un contexto de superávits sostenidos en la cuenta corriente, ha dificultado el manejo macroeconómico y amenazado la competitividad externa de la economía, al apreciarse el won coreano. Por ello, las autoridades tomaron una serie de medidas de política económica para aminorar su impacto. Éstas consistieron en primer lugar en la acumulación de reservas internacionales de parte del Banco de Corea, que culminaron en más de 262 billones de dólares antes de la crisis (gráfica 4) y su esterilización a través de emisiones de títulos públicos, con la consecuente expansión de estos mercados. En segundo lugar, se dieron a partir de 2005 medidas adicionales de desregulación de las salidas de capitales

19. Ahn, Byung Chan, "Capital flows and effects on financial markets in Korea: developments and policy responses”, op. cit., p. 306. 


\section{Gráfica 3}

Corea del Sur: flujos brutos de capitales, 1988-2007, en \% del PIB

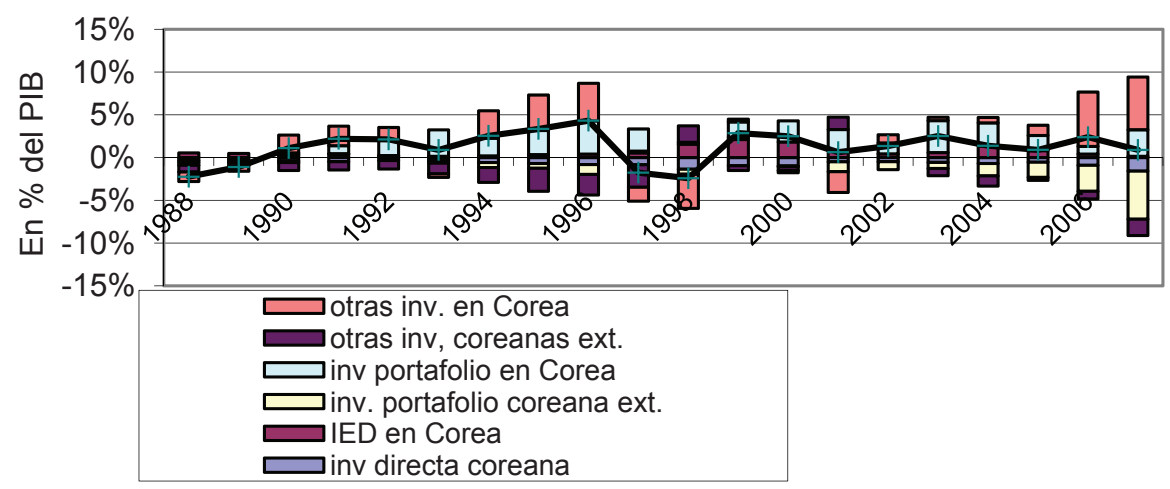

Fuente: FMI.

coreanos (inversiones de portafolio e inversión directa), ${ }^{20}$ las que llevaron a una elevación de los flujos de salida a partir de 2006, ${ }^{21}$ y a algunas medidas orientadas a frenar las entradas, en especial de fondos bancarios. ${ }^{22}$ Aun con estos dispositivos los flujos netos positivos han alcanzado en promedio $2 \%$ del PIB entre 1999 y 2007, y el won se ha apreciado a un ritmo promedio de $6 \%$ anual en el periodo 2002-2006 ${ }^{23}$ (gráfica 4).

20. Se trató en primer lugar del programa "Korea's Overseas Investment Action Plan" de mediados de 2005, que abolió límites a las inversiones de empresas no financieras y a las inversiones inmobiliarias, del "Foreign Exchange Liberalization Plan" de mayo de 2006, que liberalizó las transacciones en divisas de los residentes y de nuevas medidas en 2007 (McCauley, "Managing Recent Hot Money Inflows in Asia”, ADB Institute Discussion Paper, núm. 99, 2008).

21. Las inversiones de portafolio fuera del país han dado lugar, en forma inesperada, a entradas adicionales de fondos, pues para protegerse del riesgo cambiario, los fondos de inversión coreanos han vendido fuertes cantidades de forward de divisas, operaciones que llevaron a un incremento de la contratación de créditos en moneda extranjera en los mercados internacionales (Kim, Kyungsoo, Byoung-Ki Kim, y Young Kyung Suh, "Opening to Capital Flows and Implications from Korea", op. cit., p. 30).

22. Estas medidas se abordan más adelante.

23. Ahn, Byung Chan, "Capital flows and effects on financial markets in Korea: developments and policy responses”, op. cit., p. 314. El valor nominal del won pasó de alrededor de 1,320 won/ US\$ a inicios de 2002, después de la crisis vinculada al estallido de la burbuja de las nuevas tecnologías, a menos de 950 won/Us\$ a inicios de 2008. 


\section{Gráfica 4}

Tipo de cambio y reservas internacionales de Corea enero 1998-diciembre 2008

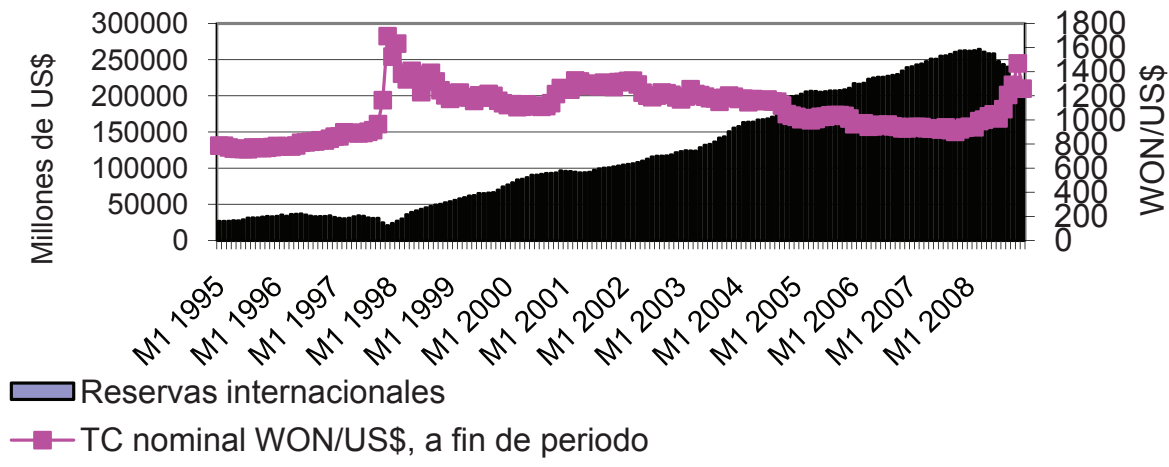

Fuente: FMI.

En el último quinquenio, periodo en el cual se han producido las entradas más masivas, la composición de los fondos se ha alterado sensiblemente. Así, la IED ha desempeñado un papel menor frente a los flujos de portafolio y en 2006-2007, en relación con las "otras inversiones" — préstamos y depósitos- bancarias: ambas categorías de flujos han influenciado notablemente el desarrollo y la trayectoria de los mercados domésticos de títulos y fueron las principales vías financieras del contagio de la crisis global en Corea.

En el mercado accionario, punta de lanza de la internacionalización financiera en el país, la inversión de portafolio extranjera ha pasado de $18 \%$ de la capitalización en 1999 a más de 37\% en promedio entre 2003 y $2006^{24}$ —y mucho más para las empresas líderes-y contribuyó a fortalecer el nivel de actividad del mercado ${ }^{25}$ y su grado de liquidez y a elevar sus precios, como lo señala el comportamiento del índice KOSPI de la Bolsa de Seúl, que pasó de

24. Como resultado de las salidas de inversiones extranjeras, esta participación había caído a $30 \%$ de la capitalización a finales de 2007 (calculado con estadísticas en línea de Bank of Korea, 2008).

25. El volumen de las transacciones, que sólo era de 12.2 mil millones de acciones intercambiadas en 1997, culminó en 2005 en 265.3 mil millones de acciones (Ahn, Byung Chan, "Capital flows and effects on financial markets in Korea: developments and policy responses", op. cit., p. 311). 
504 puntos en 2000 a 1,897 puntos a finales de 2007. ${ }^{26}$ Simultáneamente se han establecido un elevado grado de sincronización entre los movimientos del mercado accionario coreano y las bolsas estadounidenses, como lo muestra la correlación entre los índices KOSPI y Dow Jones, que pasó de -0.27 en $1995-1997$ a 0.61 en promedio entre 1999 y 2006..$^{27}$

Por otro lado, el mercado de bonos, que no fue hasta hace poco una vía importante para la entrada de capitales extranjeros, debido a la crisis que experimentó el compartimiento de bonos privados en 2003, iniciada con la quiebra del conglomerado Daewoo, se ha internacionalizado en forma muy reciente a través del segmento de bonos públicos, en un proceso encabezado por los bancos extranjeros que operan en el país, quienes ostentarían $15 \%$ del valor de los bonos públicos en circulación; como lo veremos, este proceso está estrechamente vinculado con desarrollos en los mercados de derivados financieros. ${ }^{28}$

En efecto, el desarrollo de los mercados de derivados financieros en Corea ha ido de la mano de la mayor penetración de inversionistas extranjeros y del aumento de la actividad en los mercados accionarios, cambiarios y de bonos públicos. El volumen de transacciones en opciones del índice KOSPI 200 se ha elevado significativamente entre 2003 y 2007, años que corresponden a la mayor participación extranjera en la bolsa de Seúl, y las transacciones en los mercados de derivados de divisas (forwards y swaps) han crecido a tasas anuales promedio superiores a $40 \%$ en el mismo periodo. ${ }^{29}$

En materia de flujos bancarios, Corea experimentó después de la crisis de 1997-98 disminuciones de pasivos bancarios durante varios años, mientras los bancos locales amortizaban sus deudas anteriores, seguidas de contrataciones de deudas muy moderadas hasta 2005. Sin embargo, los años 2006 y 2007 se caracterizaron por un nuevo endeudamiento de una magnitud a priori alarmante - $\mathrm{y}$ que de hecho alarmó a las autoridades coreanas-, pues entre 2005 y 2007 superó en términos tanto absolutos como relativos el endeudamiento que condujo a la crisis de 1997; en dos años la deuda externa se elevó de $23.7 \%$ hasta cerca de $40 \%$ del PIB, con casi la mitad a corto

26. Datos en línea del Bank of Korea; el índice KOSPI tenía una base de 100 en 1980.

27. Ahn, Byung Chan, "Capital flows and effects on financial markets in Korea: developments and policy responses", op. cit., p. 311.

28. McCauley, "Managing Recent Hot Money Inflows in Asia",op. cit.

29. Véase Ahn, Byung Chan, "Capital flows and effects on financial markets in Korea: developments and policy responses", op. cit., pp. 311-315. 
plazo. ${ }^{30}$ Sin embargo, el carácter de estas deudas difería netamente de aquéllas contratadas antes de 1997, por lo que hasta mediados de 2008 numerosos análisis financieros subrayaban el bajo nivel de riesgo que representaban para la economía coreana; ${ }^{31}$ no contenían los descalces o diferenciales de plazos o de divisas entre activos y pasivos que desempeñaron un papel protagónico en la crisis coreana de 1997.

Por un lado, una proporción relativamente alta de estas deudas —estimada en algo más de una tercera parte- se hallaba respaldada en un plazo de tres a cuatro años por los ingresos de exportación de los constructores navieros y otros exportadores coreanos: de hecho, estas deudas eran contratadas por bancos que cubrían una posición al ejercer de contraparte de estos exportadores en operaciones con derivados financieros (forwards de divisas) en las cuales las empresas buscaban proteger sus ingresos futuros de la apreciación del won. ${ }^{32}$ Por otro lado, una proporción también importante de esta deuda consistía en préstamos de parte de sus matrices a los bancos extranjeros que operan en Corea, por lo que se consideraba garantizada por este vínculo. En 2006 y 2007 estos fondos provenientes de los bancos matrices se dedicaron a operaciones de arbitraje destinadas a aprovechar los diferenciales de interés entre los mercados locales e internacionales, de tipo carry trade, ${ }^{33}$ que involucraban en particular los mercados domésticos de bonos públicos y de derivados financieros (gráfica 5). Por último, la fracción de la deuda contratada por bancos locales, considerada como de mayor riesgo, era menor y respaldada enteramente por activos de corto plazo en divisas. ${ }^{34}$

30. FMI, Country Report $n^{o}$ 09/262, 2009, op. cit., p. 32.

31. Dwor-Frécaut, Dominique, "Korea's money market", Korea's Economy 2008, Korea Economic Institute, 2008, pp. 21-30. Kim, Kyungsoo, Byoung-Ki Kim \& Young Kyung Suh, "Opening to Capital Flows and Implications from Korea", op. cit.

32. Al vender los exportadores forwards de US $\$$, los bancos locales que los adquirían ajustaban su posición en divisas vendiendo a su vez dólares en el mercado spot. Conseguían estas divisas vía el mercado de derivados, a través de swaps acordados con subsidiarias de bancos extranjeros.

33. Las operaciones de carry trade consisten en un endeudamiento en divisas con tasas de interés bajas - sobre todo yen o franco suizo-; los fondos así conseguidos serán invertidos en otras divisas y en instrumentos con tasas de interés más altas - típicamente en economías emergentes.

34. En contraste, el desglose de los pasivos de corto plazo del sistema bancario coreano por nacionalidad de origen de las instituciones señalaba la posición desequilibrada de las sucursales de bancos extranjeros, que concentraban $60 \%$ de los pasivos en 2007, y mostraban una relación de pasivos a activos externos de corto plazo de 8.4 (McCauley, Robert, y Jens Zukunft, "Asian banks and the international interbank market", op. cit., p.72). 


\section{Gráfica 5}

Cómo los bancos extranjeros en Corea del Sur fondean sus inversiones en bonos del tesoro coreano

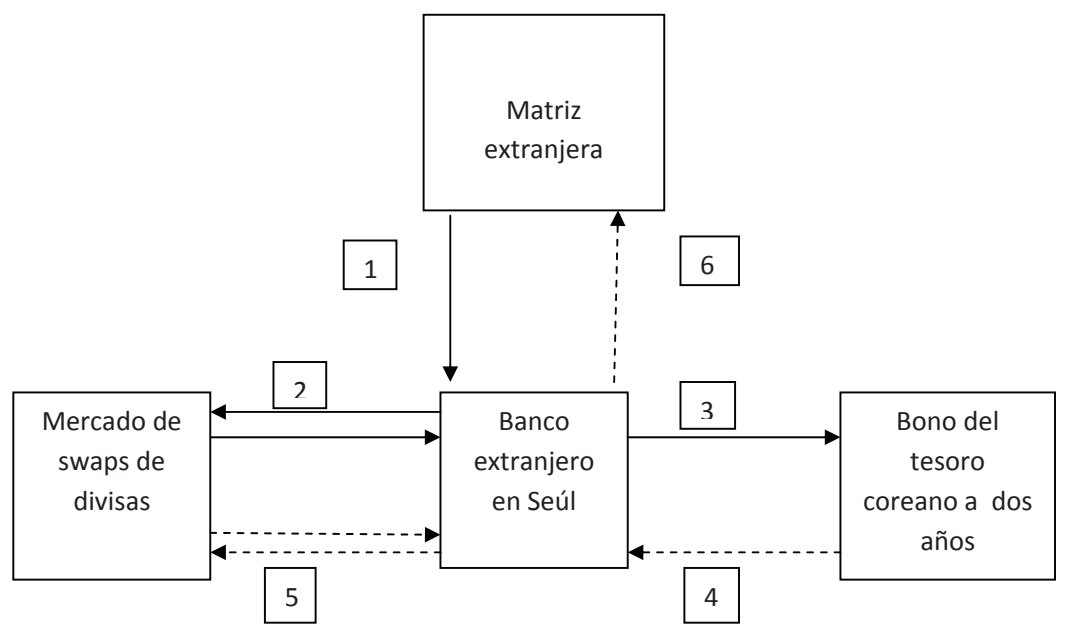

1. El banco extranjero (BE) en Seúl contrae deuda a tres meses en dólares de su matriz. 2. BE entra en el mercado de swaps de divisas, intercambiando dólares a tasa flotante por won a tasa fija. 3. BE invierte los fondos en won en bonos del tesoro coreano a dos años. 4. BE recibe tasa fija en won de su inversión en bonos del tesoro. 5 . BE paga tasa fija en won a la contraparte en el mercado de swaps de divisas, obteniendo ganancias de la diferencia entre el rendimiento de los bonos del tesoro y la tasa fija acordada con la contraparte en el swap de divisas. 6 . BE recibe tasa LIBOR a tres meses de su contraparte en el swap de divisas, y lo pasa a su matriz.

Fuente: McCauley y Zukunft, 2008.

Como lo hemos señalado, estos desarrollos suscitaron una respuesta de las autoridades coreanas, que introdujeron varias medidas y recurrieron a la "persuasión moral" para frenar las entradas de fondos y limitar la contratación de préstamos de los bancos extranjeros con sus matrices (abril de 2007), así como el endeudamiento directo de empresas no financieras coreanas en los mercados financieros internacionales, a través de la reactivación de los límites abandonados en años anteriores. Estas restricciones han sido relativamente exitosas, pues tanto el financiamiento externo de los bancos extranjeros que operan en Corea, como sus posiciones en el mercado de bonos públicos se 
han estabilizado, ${ }^{35}$ pero han sido tardías, pues se tomaron cuando la deuda ya se había elevado sensiblemente. Por otro lado, las medidas profundizaron el diferencial de rendimientos, que atrajo operaciones de otros inversionistas especulativos, como los hedge funds, que reemplazaron en parte las actividades de los bancos.

\section{El contagio de la economía coreana: ¿por qué Corea ha sido más golpeada por la crisis financiera?}

Corea experimentó algunos efectos tempranos de la crisis en forma de salidas de inversiones del mercado accionario en 2006 y 2007, y en la primera mitad del año 2008 se produjo un déficit de su cuenta corriente, debido principalmente al alza de los precios de las materias primas importadas — vinculada, en una proporción difícil de medir, a la incorporación de los commodities en las estrategias de inversión en tanto alternativa a los activos financieros-y al consecuente deterioro de los términos de intercambio del país. El impacto pleno de la crisis se dio a partir de agosto-septiembre de 2008, con el desplome de las exportaciones y el cambio de signo de las entradas de capitales.

Debido al carácter sistémico de la crisis, el escenario vivido por Corea a raíz de la masiva reversión de los flujos de capitales internacionales coincide estrechamente con lo experimentado por las demás naciones emergentes: agudo desplome del valor de la moneda nacional a partir de septiembre de 2008 (hasta alcanzar una paridad de 1,533 Won/us\$ en febrero de 2009) y de los precios del mercado accionario con el índice KOSPI a menos de 1,000 puntos en octubre-noviembre. ${ }^{36}$ De hecho, se produjeron salidas netas en las distintas categorías de flujos hasta un nivel de 5.5\% del PIB (gráfica 3).

¿En qué difirió la situación de Corea de la de otras economías de Asia del Este? En términos generales, la gravedad de la crisis coreana se relaciona con el carácter financieramente más abierto de su economía, señalado por los niveles más altos de sus pasivos externos, por el mayor peso que han adquirido las operaciones de los inversionistas extranjeros en sus mercados internos y por la más estrecha correlación entre precios de activos financieros internos y variables externas como tasas de interés o índices accionarios. Así, las salidas

35. McCauley, "Managing Recent Hot Money Inflows in Asia", op. cit.

36. Mizuho Research Institute, "The Sharp Fall of the South Korean Won in 2008: the Background and Prospects". Consultado vía http://www.rgemonitor.com, mayo de 2009. 
de fondos del mercado accionario en 2006 y 2007, debidas a la mayor aversión al riesgo de los inversionistas internacionales, y/o a su necesidad de cerrar posiciones y de asumir pérdidas en otros mercados en razón de la contracción de la liquidez mundial, ${ }^{37}$ fueron más masivas en Corea que en otras economías asiáticas debido a la mayor participación de la inversión extranjera en la bolsa de Seúl y a la liquidez del mercado, que facilitó la liquidación de activos sin que se desplomaran las cotizaciones. Por otro lado, las operaciones de arbitraje que involucraban el mercado de bonos, de poco o nulo provecho para la economía, dificultaron notablemente la política monetaria ${ }^{38}$ y expusieron el mercado de bonos públicos a la volatilidad que resultó de salidas bruscas de fondos en 2008.

Ahora bien, aun cuando las salidas de capitales del mercado accionario ocurrieron primero y fueron masivas, fue otra vez la deuda bancaria externa la que estuvo en el vórtice de la crisis. La crisis puso en evidencia el peligro asociado con la expansión de la deuda externa, así como las fallas de los análisis presentados en la sección anterior, que subvaloraron varios tipos de riesgos. Se trata en primer lugar del riesgo asociado a la refinanciación periódica de estas

la gravedad de la crisis coreana se relaciona con el carácter financieramente más abierto de su economía deudas, una operación que no planteaba dificultades en un mercado "normal" pero que se volvió muy difícil en el marco de la crisis de liquidez global, en la cual los bancos internacionales empezaron a reclamar sus fondos. En segundo lugar, si bien el nivel de la deuda externa comparado con el PIB o las exportaciones del país no era alto, su rápido incremento creó una "externalidad" negativa para la economía, debida al deterioro de la razón entre deudas de corto plazo y reservas internacionales, un indicador de vulnerabilidad financiera muy

37. Los inversionistas que más liquidaron posiciones fueron fondos de inversión a largo plazo anglosajones, así como hedge funds (Investment \& Securities, Korea Strategy. Foreign capital outflows likely to continue, 2009. Consultado en http://www.wooriwm.com, junio de 2009).

38. Kim, Soyoung, y Doo Yong Yang, "Managing Capital Flows; The Case of the Republic of Korea", $A D B$ Institute Discussion Paper, núm. 88, 2008. En este trabajo se discuten ampliamente los dilemas a los cuales se enfrentaba la política monetaria coreana. Véase también Kim, Kyungsoo, Byoung-Ki Kim, y Young Kyung Suh, "Opening to Capital Flows and Implications from Korea", op. cit. 
utilizado desde las crisis de los noventa. Ello modificó en forma negativa las percepciones de riesgo-país en los mercados financieros internacionales, aun cuando el bajo nivel del endeudamiento público y la mejoría de la situación financiera de las grandes empresas coreanas indicaban un nivel de riesgo menor al que mostraba la economía en la anterior crisis (cuadros 1 y 2 ).

\section{Cuadro 2}

Corea del Sur: indicadores de vulnerabilidad financiera externa, 2000-2008

\begin{tabular}{|c|c|c|c|c|c|c|c|c|c|}
\hline & 2000 & 2001 & 2002 & 2003 & 2004 & 2005 & 2006 & 2007 & $2008^{*}$ \\
\hline $\begin{array}{l}\text { Deuda consolidada del } \\
\text { Gobierno Central (\% del PIB) }\end{array}$ & 31.8 & 35.3 & 33.4 & 32.6 & 33.5 & 36.2 & 36.5 & 35.7 & 35.9 \\
\hline $\begin{array}{l}\text { Balanza de cuenta } \\
\text { corriente (\% lde PIB) }\end{array}$ & 2.4 & 1.7 & 1 & 2 & 4.1 & 1.9 & 0.6 & 0.6 & -1.3 \\
\hline $\begin{array}{l}\text { Reservas oficiales en meses } \\
\text { de importaciones } \\
\text { (proyección para todo el año) }\end{array}$ & 6 & 7.2 & 7.9 & 8.6 & 8.8 & 8 & 7.7 & 7.3 & 5.8 \\
\hline $\begin{array}{l}\text { Reservas/deuda externa } \\
\text { total de corto plazo }\end{array}$ & 134.1 & 170.4 & 161.4 & 194.9 & 254.3 & 239.6 & 172.5 & 130.9 & 105.8 \\
\hline $\begin{array}{l}\text { Deuda externa total/ } \\
\text { exportaciones }\end{array}$ & 71.6 & 71.3 & 73.8 & 68.4 & 57.5 & 56.2 & 68.1 & 86.5 & 89 \\
\hline $\begin{array}{l}\text { Intereses sobre deuda } \\
\text { externa/exportaciones }\end{array}$ & 3.3 & 3.1 & 2.1 & 1.4 & 1.1 & 1.4 & 1.7 & 1.9 & 0.6 \\
\hline $\begin{array}{l}\text { Apreciación det tipo } \\
\text { de cambio real } \\
\text { (base } 12 \text { meses) }\end{array}$ & 7.3 & -6.2 & 4.9 & 1.7 & 1.8 & 12.1 & 7.4 & 0.1 & $\begin{array}{l}-13.2 \\
\text { (Abril, } \\
2008)\end{array}$ \\
\hline
\end{tabular}

* proyección.

Fuente: FMI, 2008 y 2009.

Ya iniciada la crisis global, la disminución de las reservas internacionales, en medio de los esfuerzos para defender la moneda nacional, empeoró la relación pasivos de corto plazo-reservas internacionales, y subrayó el riesgo asociado a las elevadas necesidades de refinanciación de deudas de corto plazo en divisas en un contexto de aguda escasez de crédito internacional - Corea, como las demás economías emergentes o en desarrollo, no se financia en el exterior en su propia moneda sino en monedas internacionales-; ${ }^{39}$ sumando estas

39. Esta característica es el "pecado original" (original sin) de la literatura especializada (Eichengreen, Barry, y Ricardo Hausmann (ed.), Other People's Money: Debt Denomination and Finan- 
necesidades al déficit en cuenta corriente de 2008 y a las inversiones de portafolio que se podían revertir rápidamente, se obtenían requerimientos de divisas muy superiores aun a las masivas reservas internacionales del país. Esta circunstancia, al acercar el análisis de la situación de Corea del que se hacía de los países de Europa del Este, cuyas deudas externas habían crecido a ritmo acelerado en los últimos años, expuso al país a un contagio proveniente de una eventual moratoria en una región bastante alejada en muchos aspectos y le puso al borde de sufrir de una "profecía autocumplida", en la cual el temor de los inversionistas y las acciones que emprenden para protegerse causan los propios eventos que desean evitar. Cabe agregar que un elemento adicional de riesgo de contagio provino del hecho de que los mismos bancos europeos, demasiado expuestos a Europa del Este, habían prestado fondos a Corea. En consecuencia, los bancos coreanos experimentaron problemas para refinanciar sus deudas en el cuarto trimestre de 2008, bajando la tasa implícita de renovación (roll over) a 70\%, mientras el margen exigido sobre los credit default swap (CDS) de estas mismas instituciones se elevaba de menos de 200 puntos base en julio de 2008 , a casi 700 puntos en septiembre-octubre de 2008, y otra vez en febrero de 2009, señalando así el mayor nivel de riesgo percibido por los mercados internacionales. ${ }^{40}$

Estos acontecimientos recientes, y los varios episodios de crisis de los años 1980 y 1990, en los cuales fuertes entradas de fondos seguidas de un cese brusco han desempeñado un rol central, subrayan que cabe una reflexión más profunda acerca de los beneficios reales de las entradas de fondos externos para una economía como la coreana. Los beneficios que la teoría económica convencional espera de la liberalización de los flujos de capitales transfronterizos son amplios: entre los beneficios directos, se espera una mejor asignación agregada de los recursos al fluir los capitales hacia los mejores proyectos, social y económicamente más rentables en el plano mundial; la complementación con ahorro externo del ahorro interno de las economías en desarrollo, que podrían así elevar sus tasas de inversión emprendiendo más proyectos de alto rendimiento para alcanzar ritmos de crecimiento más altos; la amortiguación de los "choques" negativos al ingreso y al consumo gracias a la disponibilidad

cial Instability in Emerging Market Economies, University of Chicago Press, Chicago, 2005.

40. Kato, Takatoshi, "Why Asia Has Been Hit So Hard by the Global Economic and Financial Crisis?”, presentación en el Eighteen General Meeting of the Pacific Economic Cooperation Council, mayo 2009. Consultado en http://www.imf.org, el 19/09/2009. 
de financiamiento que permitiría absorberlos paulatinamente, y el mejor reparto de los riesgos a través de la diversificación internacional de las carteras. Indirectamente, se esperan asimismo mejoras a la eficiencia de las instituciones financieras internas de las economías y el desarrollo de sus mercados financieros internos bajo el impulso de los inversionistas extranjeros.

Si la apertura de la cuenta de capitales resultó controvertida desde sus inicios en los años 1970 y 1980, carácter que se reforzó con las crisis de los años 1990 en las economías emergentes, desencadenadas por episodios de entradas excesivas seguidas de salidas en masa, ${ }^{41}$ en años recientes se han producido, desde diversos enfoques teóricos, trabajos que cuestionan la capacidad de tales flujos para financiar en el largo plazo un proceso de desarrollo consistente. ${ }^{42}$ Por otro lado, el análisis de las características empíricas de los masivos flujos de los últimos años, en especial de aquéllos recibidos por las economías asiáticas de alto crecimiento como Corea, sugiere que éstos se han orientado poco hacia la inversión productiva y por ende hacia el crecimiento económico; ${ }^{43}$ procíclicos y desestabilizadores, responden ante todo a acontecimientos y a variables de corto plazo en las economías desarrolladas. P. Artus $^{44}$ señala que la liberalización de los flujos de capitales no ha traído los resultados esperados: en particular porque los flujos corporativos, como la IED, más estrechamente vinculados con la inversión productiva y con mayor capacidad de fomentar ganancias en eficiencia, son minoritarios frente a la enorme burbuja de los flujos de portafolio, que buscan ganancias estrictamente financieras o especulativas en los mercados de títulos negociables o a través del carry-trade, o frente a los flujos que han financiado la especulación inmobiliaria. En Asia, los flujos recibidos por la región en los últimos años han sido cada vez más volátiles y procíclicos; así, en coyunturas de declive global los inversionistas internacionales "no actúan como si aceptaran su parte de la

41. French-Davis, Ricardo, Crisis financieras en países "exitosos", CEPAL, 2001.

42. Kregel, Jan, "The Global Crisis and the Implications for Developing Countries and the BRICs", Public Policy Brief, núm. 102, The Levy Economics Institute at Bard Collage, 2009,; Kregel, Jan, "External Financing for Development and International Financial Instability", G-24 Discussion Paper, núm. 32, UnCTAD, octubre de 2004; Bresser-Pereira, Luiz Carlos, y Paulo Gala, “¿Por qué el ahorro externo no promueve el crecimiento?, Investigación Económica, vol. LXVII, núm. 263, enero-marzo, 2008: 107-130.

43. Baek, In-Mee, "Portfolio investment flows to Asia and Latin America: pull, push or market sentiment?”, Journal of Asian Economics, vol. 17, núm. 2, 2006: 363-373.

44. Artus, Patrick, "International capital flows are more destabilizing than efficient", Flash Economics, núm. 102, Natixis, 10 de abril de 2009. 
pobre cosecha [...] sino que se precipitan hacia la salida", ${ }^{45}$ creando volatilidad en los mercados accionarios y cambiarios locales. Finalmente, la abundancia de la liquidez global ha llegado a desvincular el valor de los activos financieros de los valores fundamentales de la economía real, y a que respondan casi exclusivamente a fluctuaciones en el "sentimiento" de los inversionistas.

La experiencia reciente de Corea resulta ilustradora acerca de los beneficios y de los costos que ha aportado la apertura financiera a la economía del país. La apertura ha aportado beneficios microeconómicos indirectos que consisten sobre todo en el fortalecimiento de los mercados domésticos de títulos negociables y de derivados financieros, dinamizados por una elevada participación extranjera, y en la introducción de innovaciones y prácticas financieras internacionales por parte de las instituciones extranjeras que operan en Corea. Ha permitido también la diversificación internacional del portafolio de los inversionistas coreanos. En contraste, en el plano macroeconómico los costos parecen haber predominado: la inversión productiva no ha registrado una trayectoria más destacada en los últimos años, caracterizados por masivas entradas de fondos, y la magnitud misma de estos fondos ha dificultado mucho las políticas macroeconómicas, inhibiendo en particular la política monetaria. A la vez, al estar ahora estrechamente conectados los mercados financieros domésticos del país con los mercados internacionales, la apertura ha agregado una nueva modalidad de contagio muy poderosa a través de la cual las crisis externas impactan más rápidamente que a través de la vía convencional de las exportaciones de bienes. El mercado accionario en 2006-2007, el mercado de bonos públicos y la deuda bancaria en divisas en 2008 constituyeron los tres vectores de contagio financiero de la economía coreana. Finalmente, los riesgos de la apertura son magnificados por la posición de "mercado emergente", de destino atractivo con rendimientos más elevados, que ocupa Corea en la esfera financiera global, pues esta condición expone a la economía a entradas excesivas de fondos cuando existen "apetito para el riesgo" u oportunidades de arbitraje, y a salidas brutales motivadas no sólo por acontecimientos locales sino por un alza de la "aversión al riesgo" o por modificaciones en las variables financieras en los países de origen de los flujos. ${ }^{46}$

45. McCauley, "Managing Recent Hot Money Inflows in Asia", op. cit., p. 5.

46. Al respecto, cabe notar la diferencia de apreciación de riesgo asociado a una economía como la coreana frente, por ejemplo, a Australia, una economía desarrollada que presentaba niveles 


\section{Conclusiones}

Después de la crisis asiática Corea eligió reformar su modelo de desarrollo e introducir mayor libertad de mercado, en particular en el ámbito financiero. La apertura de la cuenta de capitales coreana ha sido acompañada de masivos flujos de capitales hacia el país, y más recientemente de salidas de capitales coreanos. En términos macroeconómicos, los flujos netos positivos que ha recibido el país han contribuido poco a la inversión productiva y a la modernización del país, emprendidos básicamente por agentes nacionales. Simultáneamente, han dificultado el manejo macroeconómico, creando apreciación de la moneda, pérdida de competitividad y menor efectividad de instrumentos de política económica, en especial en materia monetaria. Frente a estos costos macroeconómicos, las aportaciones de estos flujos en materia de diversificación de riesgos y de desarrollo financiero - desarrollo de los mercados internos de acciones o de bonos, o crecimiento, por ejemplo, de los mercados de derivados financieros- parecen menores o incluso ambivalentes, pues también han contribuido a potenciar la vulnerabilidad financiera externa del país.

La crisis experimentada por Corea en 2008 difiere mucho de la crisis de 1997. Esta vez no se puede atribuir el contagio de Corea a prácticas poco ortodoxas del sistema financiero interno, si bien éste presenta riesgos en materia de endeudamiento de los hogares y de las pymes. En el plano financiero, la crisis sistémica externa ha golpeado a Corea a través de las salidas de inversiones de sus mercados de títulos domésticos altamente internacionalizados y de las dificultades para renovar préstamos bancarios de corto plazo. Entre estos diferentes canales de contagio financiero, es otra vez la cuestión de la deuda bancaria externa lo que ha creado las mayores dificultades a la economía coreana.

Las intervenciones públicas masivas en las principales economías desarrolladas y en el plano nacional, las medidas adoptadas por el gobierno coreano $^{47}$ han evitado que "lo peor" — un encadenamiento fatal de quiebras

de endeudamiento interno y de deuda externa mucho más elevados —esto si en buena parte están denominados en la moneda nacional.

47. Aparte de la disminución de las tasas de interés de corto plazo, el banco central coreano ha tomado varias medidas para proveer el sistema de liquidez interna, evitar una contracción del crédito y soportar la solvencia de las instituciones (Fondo de Recapitalización Bancaria). En lo que concierne a la liquidez internacional, destacan los swaps de divisas con Estados 
corporativas y soberanas - ocurriera: varios meses después del pánico internacional de finales de 2008 los mercados financieros globales se han estabilizado y el alza de los precios de los activos pareciera incluso sugerir un retorno a la "normalidad" anterior a la crisis, con una disminución de la "aversión al riesgo" que ha propiciado el regreso de inversiones especulativas hacia los mayores rendimientos ofrecidos por los mercados emergentes; de nueva cuenta, estos flujos han vuelto a elevar los precios de los activos y a apreciar el valor de sus monedas, aun cuando las variables reales apenas han empezado a recuperarse.

En el contexto de poscrisis en el cual las estrategias de desarrollo van a tener que redefinirse, y reemplazar en buena parte la demanda internacional por demanda interna, Corea enfrenta varios retos. Por un lado, tanto los hogares coreanos como las pymes, que aportan la mayoría de los empleos, salieron sobreendeudados de la década que acabamos de analizar. Por otro, en materia de flujos de capitales el país tendrá que definirse entre mantener su posición actual favorable a la libertad de flujos, un ingrediente esencial de la estrategia que busca hacer de Corea un hub financiero para Asia del Este, y adoptar políticas más cautelosas que busquen fomentar el desarrollo de otras fuentes de demanda internas -inversiones orientadas hacia una "economía más verde" por ejemplo, o redistribución de ingreso que permita estimular el consumo de ciertas categorías de la población-, a la vez que permitan aislar al país de los flujos de "dinero caliente" y evitar así "que la estrategia de desarrollo [...] [se deje llevar por] las fluctuaciones inducidas por gobiernos extranjeros y por las condiciones monetarias internacionales". ${ }^{48}$

Unidos, China y Japón, y la provisión de divisas a los bancos que no pudieran refinanciar sus préstamos. Véase Bank of Korea, The Bank of Korea's Policy Response to Stabilize the Financial and Foreign Exchange Markets, 2009; consultado en http://www.bok.kr. En la esfera real, el gobierno coreano ha lanzado varios programas de estímulo a la demanda pública y privada.

48. Kregel, Jan, "The Global Crisis and the Implications for Developing Countries and the BRICs", op. cit., p. 13. 


\section{Series \\ Pacific Rim: \\ Globalization, Regionalization, and Domestic Trajectories}

Under the general supervision of Melba E. Falck and Arturo Santa Cruz and edited by Universidad de Guadalajara and University of Technology Sydney
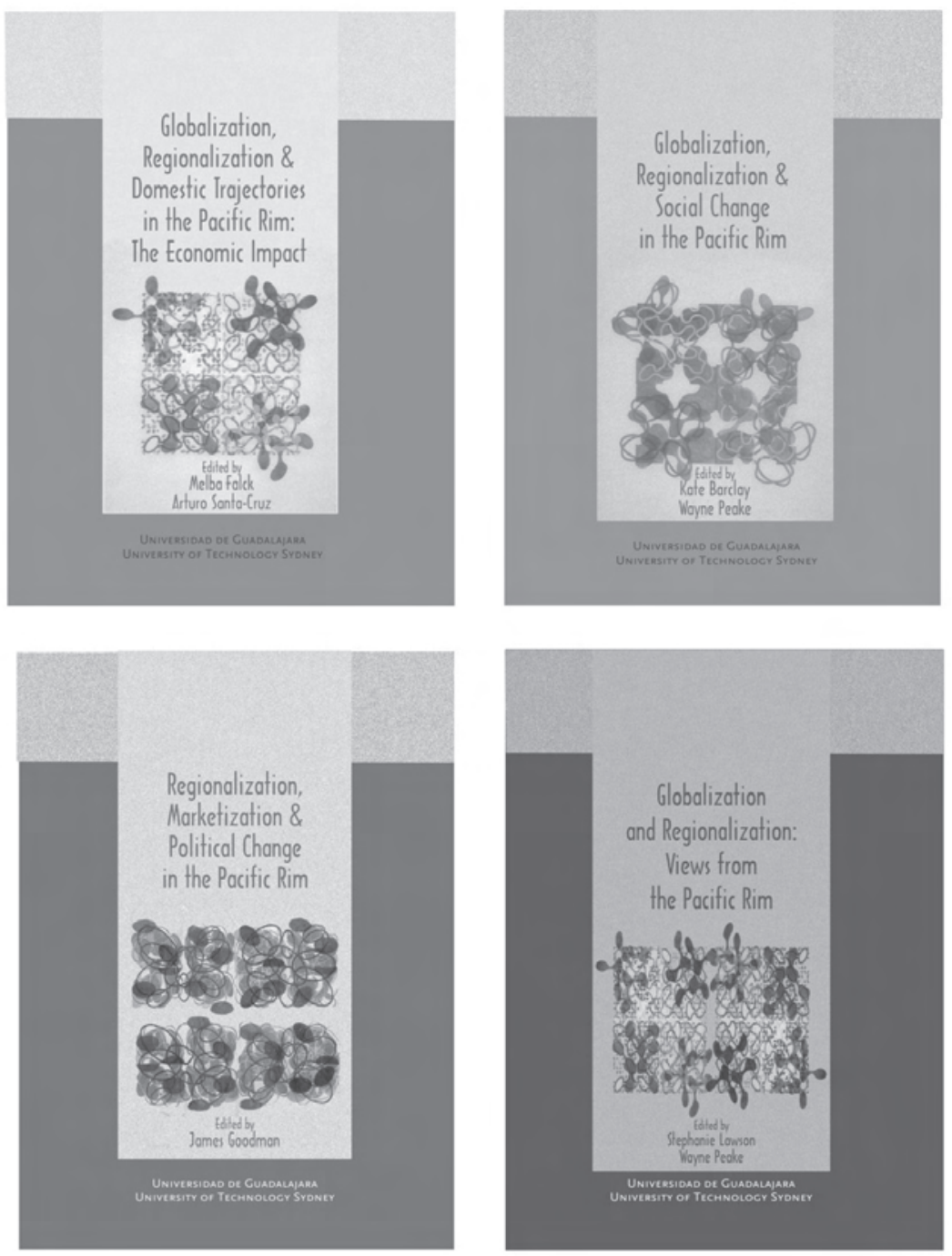